\title{
Macintosh statistical packages
}

\author{
RICHARD S. LEHMAN \\ Franklin and Marshall College, Lancaster, Pennsylvania
}

\begin{abstract}
Fifteen statistical packages for the Apple Macintosh computer are reviewed, and their features are listed and compared. Many do not follow the Macintosh user interface and are likely to be confusing to novices. Accuracy tests are reported for several programs.
\end{abstract}

As of the fall of 1985, all students entering Franklin and Marshall College are strongly encouraged to purchase an Apple Macintosh personal computer. This recommendation is part of the college's decision to incorporate computing throughout the curriculum; the college has also provided a Macintosh to each faculty member, has greatly increased staff support for computing, and is enlarging its offerings in computer science. This report began as an in-house survey of existing Macintosh statistical programs. With the support of the college's Center for Academic Computing (which made use of funds provided by the Sloan Foundation under its "New Liberal Arts" program), we purchased one of each known Macintosh statistical program.

The programs may be classifed into two major groups, although the dividing line is not altogether clear. In the first group, there are nine programs whose user interface is Mac-like to a greater or lesser degree. Some of these programs have been designed and written originally for the Macintosh; others are rewrites of older programs (ported from IBM PCs or other computers and made to fit into the Macintosh operating environment). All of these programs offer at least some degree of mouse/menu kind of control, although several have a distinctly non-Mac feel to them. Programs in the second group vary much more widely in features and capabilities but tend toward the power-use end of the spectrum; the major characteristic of these programs is that they make no effort to follow the Macintosh interface standard, but instead offer a PClike interface. The programs in this group are probably better suited for serious research use than for classroom purposes; students accustomed to a Mac interface will feel extremely frustrated with the PC-like interface. (But if the user must have mainframe power and can put up with a mainframe command system to obtain it, SYSTATone of the Group 2 programs-offers what no other program can even approach as a worthy substitute for BMDP, SPSS, or SAS.) The two groups of programs will be considered separately.

In addition to its unique user interface, the Macintosh is best known as a marvelous graphics machine. The true Mac statistical packages tend to take far better advantage of the computer's graphics capability than do the conver-

Please address reprint requests to: R. S. Lehman, Department of Psychology, Franklin and Marshall College, Lancaster, PA 17603. sions, although some converted programs do well on graphics as well. The graphics features of the programs deserve comment throughout the comparisons.

\section{DESCRIPTION OF FEATURES}

Table 1 lists the Group 1 programs and conveys a great deal of detailed information. The criteria rated in the table are described in this section. An entry of "???" indicates that the information was not available in the documentation or program, and that the publisher did not respond in time to have the information included in this survey. A list of all of the programs rated and the publishers' addresses is presented in Table 2.

\section{Capacity}

The variables listed under capacity serve to describe the programs in several ways. Many programs have two sets of limits, one for $128 \mathrm{~K}$ machines and the other for $512 \mathrm{~K}$. Some give no specific limits, but provide examples of numbers of data points or complex formulas relating capacity to data types, variables, and cases. Most programs do not explicitly recognize "grouped" data, but rely on some form of coding variable to differentiate among ANOVA or $t$ test groups. "Missing data" refers to a program's having a special way to code missing scores.

\section{Data Entry}

Several programs do not have data entry provisions, so an ASCII file must be prepared separately. "File input" indicates the kinds of files that a program can use for input; some are able to input an externally prepared ASCII file, whereas others can read only files that they have saved. "By variables" and "By cases" refer to the order of data entry: whether all cases of a variable or all of a case must be input at one time. "Yes" for both categories indicates that either can be done. "Spreadsheetstyle" means that the data entry is accomplished as it would be on a spreadsheet (e.g., StatView looks exactly like Multiplan). The ability to accept data from the clipboard facilitates moving data from other programs.

\section{Editing and Selecting}

"By case" and "By logical expression" refer to the manner in which specific cases may be selected for in- 
$\stackrel{-}{\stackrel{5}{\Xi}}$

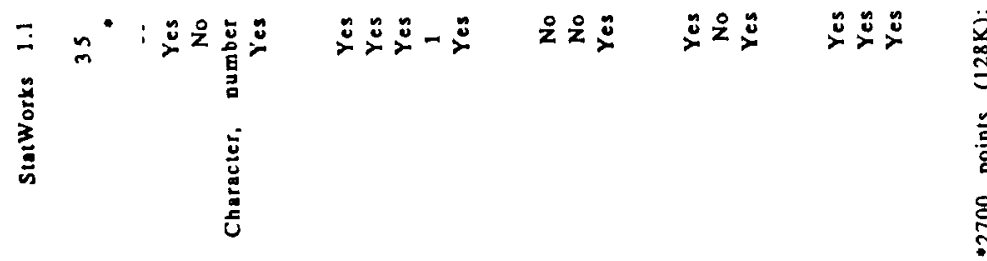

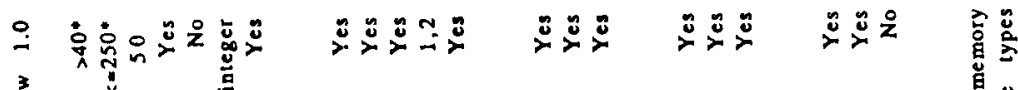

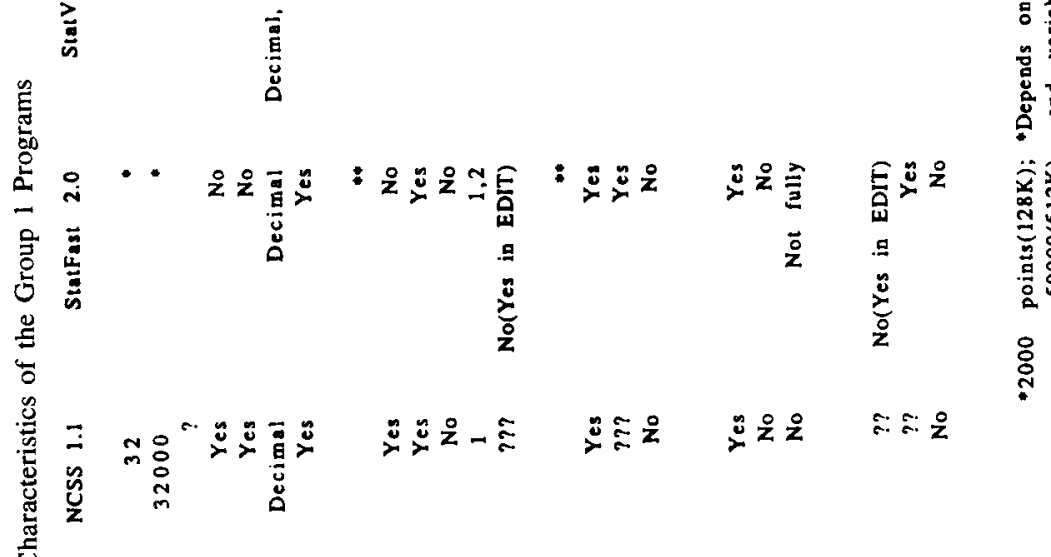

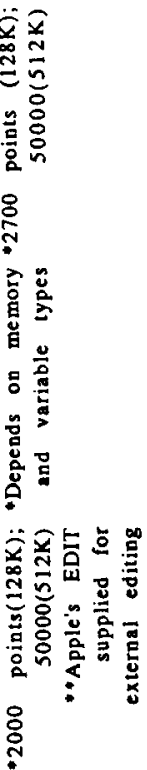

㱐

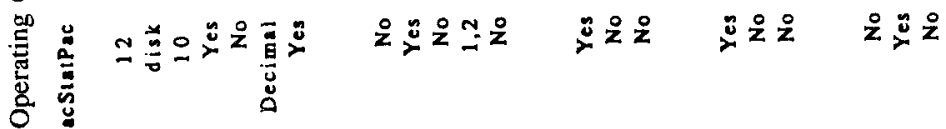

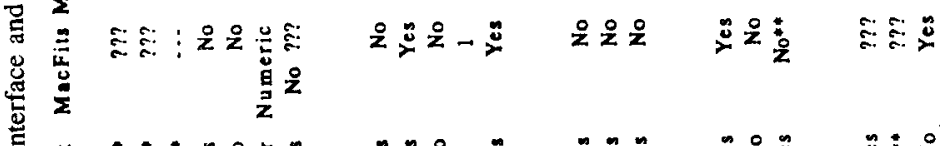

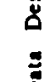

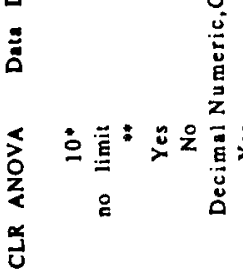

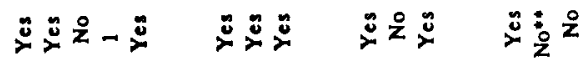

衰密

$\therefore 8$

เด

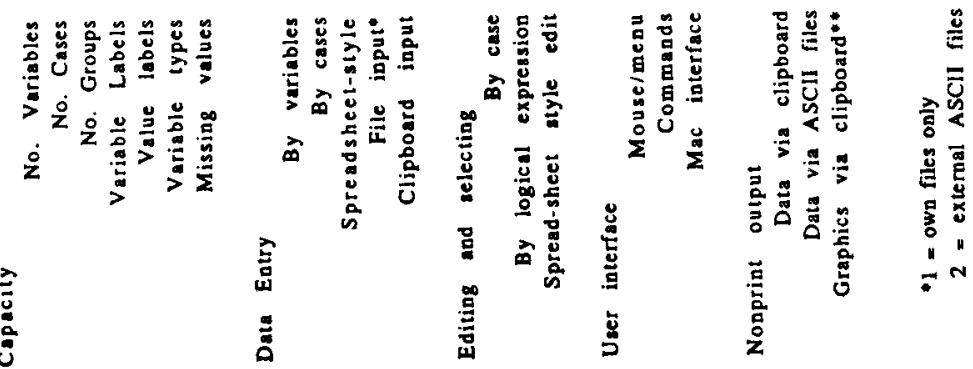



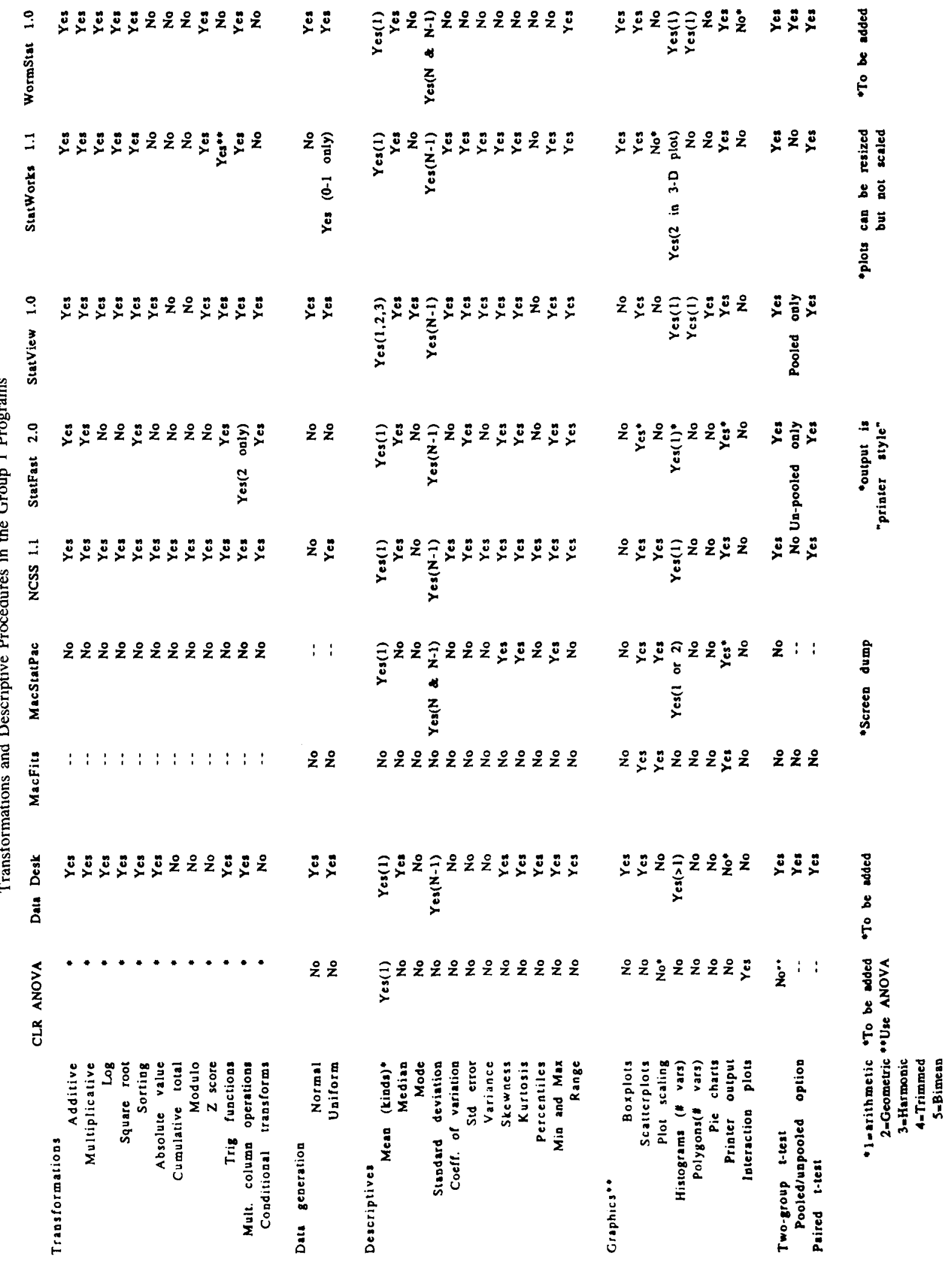


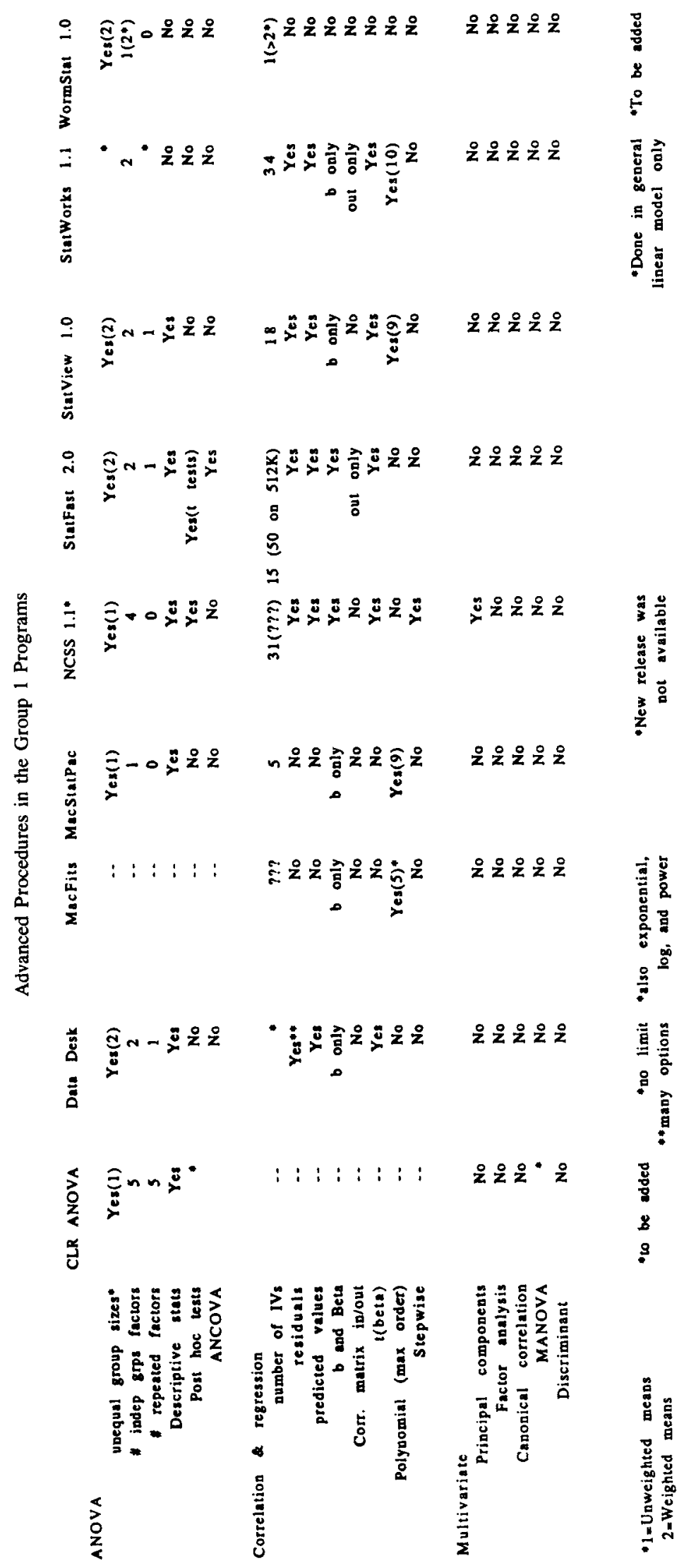




$$
\text { تٕ }
$$

:

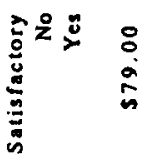

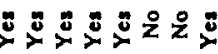

음

울요우

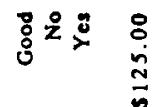

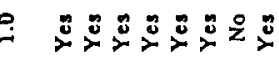

离

$\underset{\substack{n \\ \infty}}{\substack{x \\ \hdashline}}$

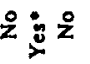

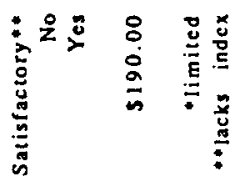

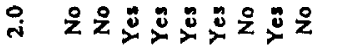

总

N妾

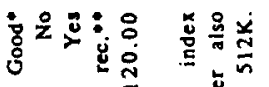

竞

-

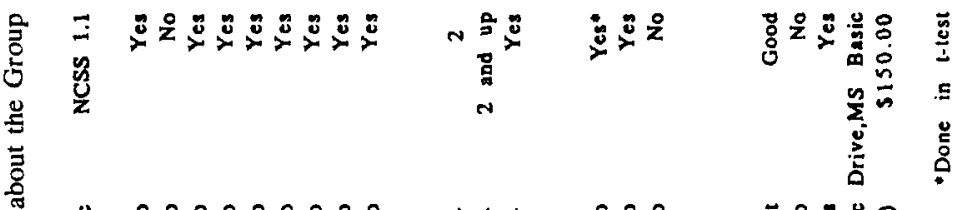

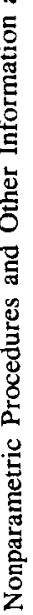

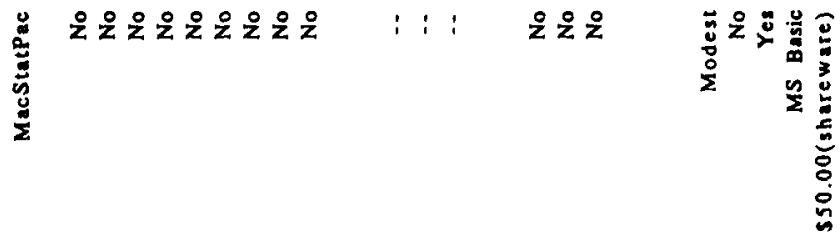

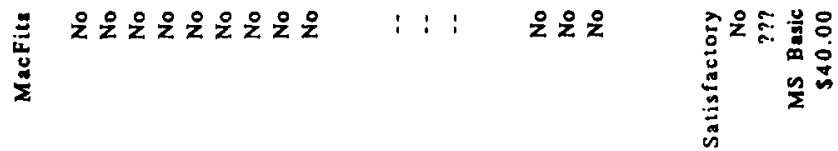

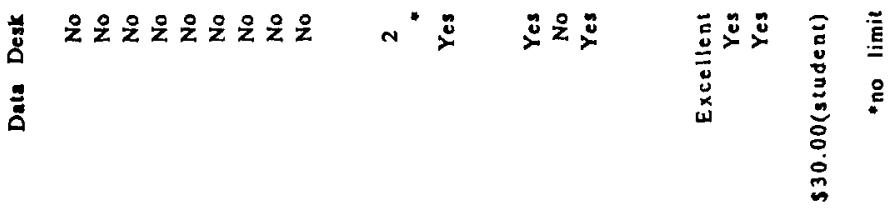

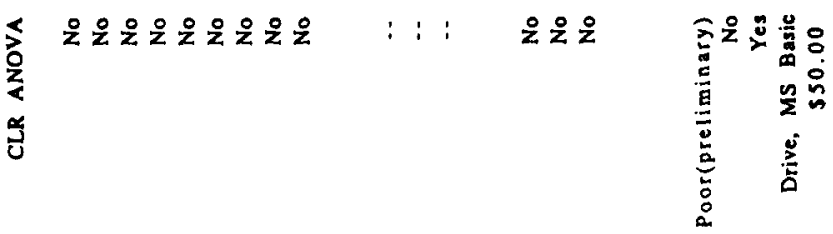

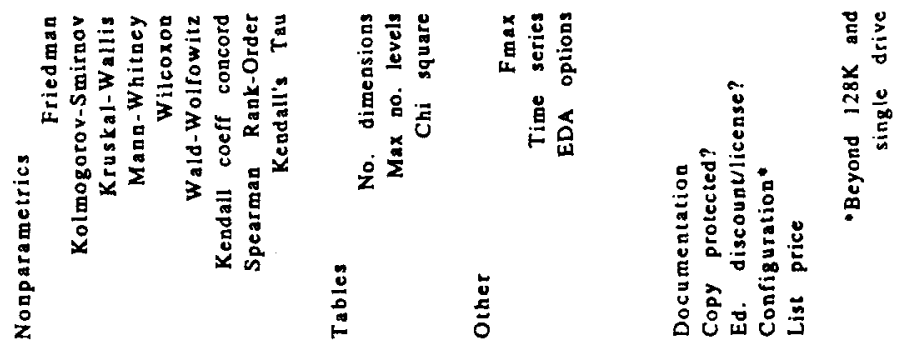


Table 2

Macintosh Statistics Programs Surveyed

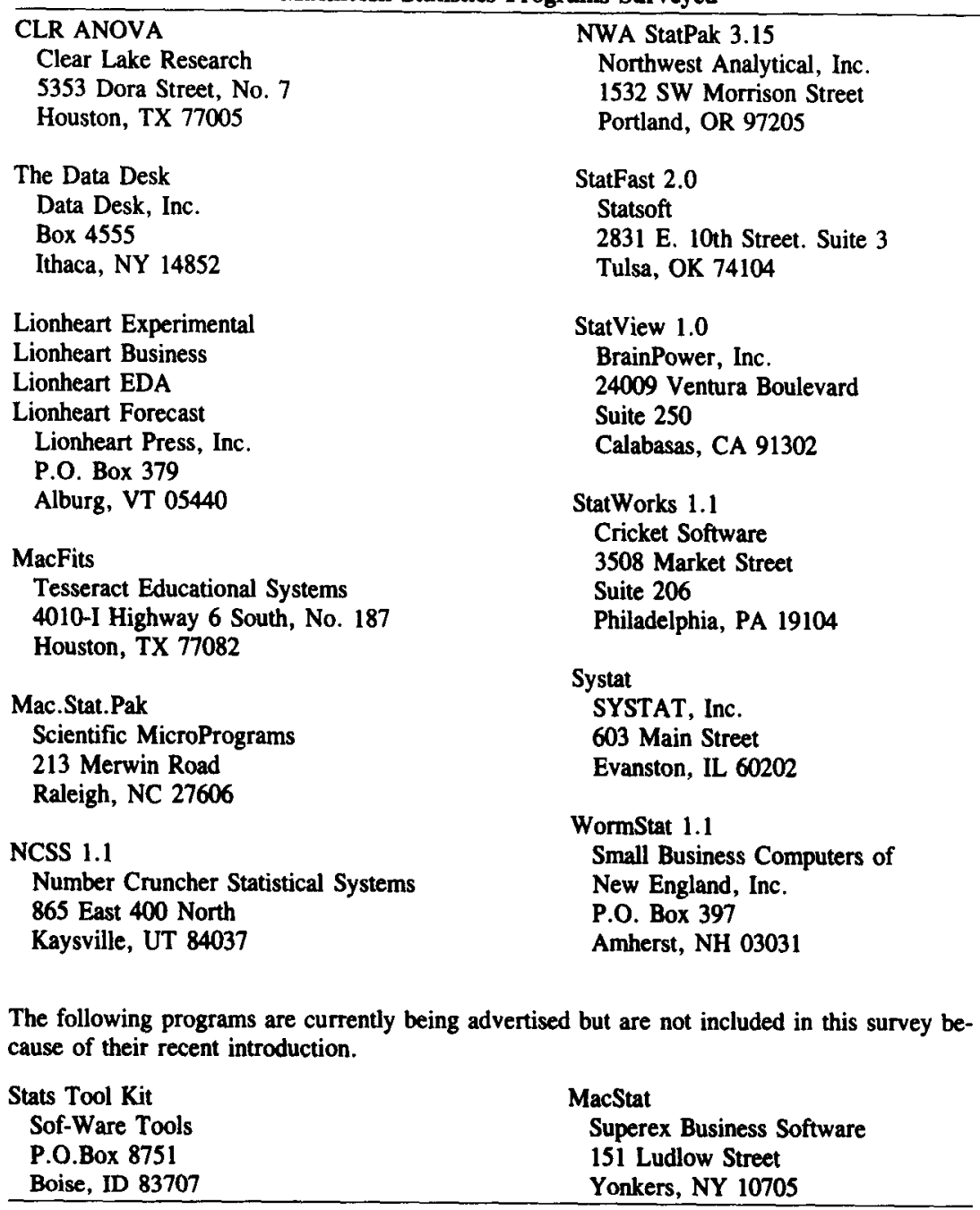

clusion in an analysis. Programs rated "No" in "Spreadsheet style editing" require the user to enter a case and/or variable number in order to change a value; "Yes" means "click on it and change it."

\section{User Interface}

These items describe how Mac-like the interface seems to be. Several of the programs appear to have been designed specifically for the Macintosh (Data Desk, StatView, StatWorks, and WormStat), whereas others (NCSS, StatFast) are modifications of programs originally written for other computers and translated with varying degrees of success to have the Macintosh style of interface.

All of the Group 1 programs use menus. Many are pulldown menus, although for some (such as StatFast), the number of the menu choice must be typed, or a return or backspace key is used to indicate "yes" or "no" to offered choices. Several programs (only StatView in Group 1) also offer keyboard commands for experienced users.

\section{Nonprint Output}

Several programs allow data to be output to the clipboard via the usual mark-and-cut/copy operation, and many also permit the creation of standard ASCII text files. Those that create ASCII files follow the usual Mac convention of tab delimiters between fields with a return at the end of a record. Two programs allow the capture of their graphics onto the clipboard, and from there to a Scrapbook or to MacPaint. In addition, most programs permit the capture of a screen image to a file (via Command-Shift-3) for later MacPaint editing. Data Desk uses a special FLASH PICTURE command to accomplish the same end.

\section{Transformations}

The most popular transformations are listed here, but some programs offer more. The Data Desk is notable for the kinds of transformation operations that it allows. "Conditional" refers to the program's ability to apply a transformation selectively, based on some logical condition. 


\section{Data Generation}

The ability to generate data, preferably both uniform and normal, is an important feature in any program to be used for teaching. Good data generation capability along with a good set of transformations can provide a resource for creative statistics instruction.

\section{Descriptives}

The most common statistics are listed here; some programs expand on this list. Programs offering several choices of mean are noted.

\section{Graphics}

The Macintosh is a superb graphics machine, and several of the programs make use of its capabilities very well. This section notes the aspects of the data that can be presented graphically.

\section{$t$ tests and ANOVA}

All programs, except the special-purpose MacFits, offer $t$ tests and at least one-way analysis of variance. Various other forms of ANOVA can be computed by defining dummy variables for multiple regression (Data Desk offers a PATTERNED DATA command for that purpose); however, only standard ANOVA designs are noted here.

\section{Correlation and Regression}

"Residuals" and "Predicted" indicate whether the program will generate and store those values for later analysis. For those programs that do not, the values can usually be computed using transformations. Any program with suitable transformations can be made to compute polynomial regression; "Yes"' indicates that the polynomial regression is available directly. Likewise, any program can do a "Stepwise" selection by conducting repeated analyses with different variable selections; "Yes" indicates that a stepwise analysis can be run automatically.

\section{Multivariate Analyses}

Clearly, the programs in this group are not intended for multivariate analyses (but see SYSTAT in Group 2).

\section{Nonparametrics, Tables, and Other}

The listing of nonparametric offerings should be selfexplanatory, as should the items listed in the "Other" category. Given properly structured data, several of the programs can form frequency tables in two dimensions. The limits are given. All of those programs compute a chi-square statistic; some offer other contingency statistics as well.

\section{Remaining Categories}

The "Documentation" entry is subjective but attempts to communicate effectively. All of the publishers who have responded have indicated at least a willingness to talk about educational discounts, many with the understanding that software for a student in a course for which purchase is required should be "about the price of a textbook." In cases in which both a "student" and a "professional" version of the program is offered, the list price quoted is based on the least expensive version. Many programs are available at substantial discount through mailorder sources.

\section{PROGRAM OVERVIEWS}

This section of the paper presents capsule summaries of the programs. For specific information on program details, consult the summary table which provides extensive detail on the programs in Group 1-those programs that generally try to follow the Mac interface standard. The preceding section defined the terms used in the table to describe the features of the programs. Table 1 presents general operational data about each program, information about the programs' elementary statistical operations, information on more advanced statistical procedures, and some additional information about each program. The table covers only the Group 1 programs; information about Group 2 is presented below.

\section{Group 1: Mac-Interface Programs}

These programs offer a wide range of the most common facilities. There are two special-purpose programs included in this group, MacFits and CLR ANOVA. Each serves one function, curve fitting and the analysis of variance, respectively. The balance of the programs are truly general-purpose statistical packages. All are characterized by some degree of "standard" Macintosh control. In some, the Mac standard user interface is thoroughly integrated; in others, the use of the various Mac features is limited, perhaps with no pull-down menus, but with mouse-activated selection from standard menus, for example.

CLR ANOVA. This package does only one thing, but does it exceptionally well. To do anything beyond the most basic forms of one- or two-way ANOVA on a Mac, this program is required. It is the only program reviewed that will construct interaction plots; when the anticipated post hoc testing procedures are added, it will be virtually unbeatable. The major fault of the program is the need to use an external data editor, a flaw that the program's originator is trying to correct. In the present version, setting up CLR ANOVA and Apple's EDIT in a Switcher rotation makes things quite workable. The only other fault of the program is that it, and its documentation, is still unfinished. The current documentation can best be described as sketchy but marginally adequate. (On the other hand, with multiway ANOVAs, the user may not need a great deal of hand holding.) When the planned additions are completed, this program alone may prove reason enough for a productive research laboratory to buy a Macintosh. It simply has no equal in doing analysis of variance computations and follow-up procedures.

The Data Desk 0.96. (This review is based on the prerelease version.) In its present form, Data Disk 0.96 (a Cornell University product) is an excellent teaching 
package, offering many options for elementary data analyses. Alone among the packages, it incorporates numerous EDA commands. Its multiple regression analytic features are another major strength, as are its extensive capabilities in generating and transforming data. The manual is by far the best for students. The program's weaknesses include a nonstandard "desktop" and manner of handling files. In addition, the screen quickly becomes cluttered with windows and variables, since every operation seems to produce a new document or variable, and many must be thrown away lest the Finder (or the user) become overloaded. Results of analyses may be placed into "bundles" (similar to file folders in the Finder), and entire bundles disposed of at one time, but the procedure for directing results to a bundle is cumbersome and easy to overlook. After a period of time, the annoyances become minor, and the program is considered a very powerful statistical tool.

MacFits. The MacFits package does nothing but curve fitting, and it does it very nicely. Five types of regression are available-linear, exponential, logarithmic, power function, and polynomial (up to order 5). The weaknesses of the program include the data editing facilities, which are awkward to use and confusing at first, and the superficial and sketchy documentation. Once the user becomes accustomed to its somewhat nonstandard user interface, the program is exceptionally easy to use, and its graphics are marvels of clarity.

Mac.Stat.Pac. This is an interesting statistical package. The program is distributed as shareware, and its users are urged to contribute modifications to the program for distribution in later updates. The program runs under Microsoft BASIC and is fully listable and modifiable. This program would not be suitable for teaching elementary statistics, but many research labs might find it to be just what they need due to its data handling and graphics capabilities. (Its three-dimensional histogram is very impressive.) The limitations are clear from the tables, but it has the core of a potentially impressive package.

NCSS 1.1 (Number Cruncher Statistical System). This may have been the first IBM PC package translated and marketed for the Mac. Unfortunately, the reviewed version of the program betrays its origins in its generally non-Mac interface and crude graphics. But there is a great deal to like in NCSS as a program for research. It is a powerful and very easy to use statistical system. It offers a number of features that other programs do not (e.g., principal components analysis, and up to four-way ANOVA with post hoc tests). A new and completely rewritten (for the Mac) version is due soon.

StatFast 2.0. This program is in its second release for the Macintosh and now operates much more like a Macintosh program is expected to behave. Its PC history is still evident in the editing and option-selection functions, some requiring typed menu choices to be acknowledged by pressing the return key. The program is cumbersome in its editing and, on a $128 \mathrm{~K}$ computer, very slow; it seems to read and write the data file before and after each oper- ation. The program comes closer to living up to its name on a $512 \mathrm{~K}$ computer, where data seem to be kept in RAM after being initially read. Students accustomed to a standard Mac interface will find StatFast frustrating to use. The program is supplied with two data editors, one internal and one external. The internal editor operates on a case and variable basis: to edit a value, the case and variable number must be supplied. The external editor is Apple's EDIT, a very fast ASCII file handler. EDIT allows clipboard input, so that it is possible to transfer data into StatFast from other applications, but only through EDIT. The program has an annoying habit of printing its opening screen on the printer, whether you want it or not. Normally, StatFast output can be directed to a file for editing or printing later, but doing so causes plots to appear "printer style," although they were in high-resolution graphics on the screen. Some output will scroll off the top of the screen during operation, making it imperative to have the printer file activated for any but the shortest operations. StatFast is actually two separate programs. One program does the basic analyses; however, to do any of the three kinds of operations on its "ADVANCED" menu (multiple regression, multifactor ANOVA/ANCOVA, repeated measures ANOVA), the user must boot a new program from a separate disk. An alternative is to set up the two programs in Switcher rotation, but to do so, the very slow $128 \mathrm{~K}$ version of the program must be used.

StatView 1.0. This is one of the most Mac-like of the packages. Editing is done in a window where all of the Multiplan controls (return, shift-return, etc.) operate perfectly. Output appears in windows, and everything is controlled from pull-down menus. As with many true Mac interface programs, the manual is an aid rather than a requirement, offering good start-up guidance, shortcuts, and suggestions on how to run the more complex analyses that are not obvious from the menus. A novice can probably boot the program and begin work with no assistance from the manual. An extensive array of transformation, selection, descriptive, and analytical features make this a very useful and flexible program. It would be a good classroom tool, and would be useful for research as long as high-level ANOVA was not required.

StatWorks 1.1. This is another truly Mac-like program. Its editing, while not a carbon copy of Multiplan's, is very smooth, simple, and intuitive. The range of features is extensive, impressive, and easy to use. Like StatView, the manual is an aid but not an initial requirement; the program's user interface and menus allow the beginner to jump in easily. The program's graphics are particuarly well done. An especially nice feature copies any active window, including graphics windows, to the clipboard for transfer to other programs such as MacPaint. Regression is very strong on StatWorks; ANOVA is somewhat less so. The only two important weaknesses of StatWorks as a teaching program are the lack of a normal data generator, and the cumbersome way that subgroups must be selected for analysis. Selection is done by choosing a sub- 
set of data-perhaps representing a single group in an ANOVA design-and copying it to a new column where the analysis is completed. In contrast, StatView keeps the whole dataset intact and conducts an analysis of a subset defined by a logical expression. This limitation will be overcome in a future release of the program.

WormStat 1.0. This program comes from Dartmouth College; an early version was widely distributed under the mistaken impression that it had been placed in the public domain. The program is intended primarily as an elementary teaching tool; its limitations on variables and cases, as well as in regression and ANOVA, would prove a severe constraint to a research worker. But as an easyto-use, low-level program, it has no equal. The user is confronted with a MacPaint-like screen with a list of tools on the left side; selecting a tool causes the analysis to be carried out on the data chosen in a variable selection window. A unique feature of the program allows the user to draw a regression line "by eye"; WormStat leaves the line on screen during its own plotting, to be compared with the program-derived formula and plot. Planned additions to the program will bring it into competition with some of the more full-featured programs.

\section{Group 2: The Non-Mac Programs}

These packages are all conversions from other computers, and none make much effort, if any, to incorporate the Macintosh user interface. Graphics and other output tend to be printer style, whether directed toward the screen or the printer.

The programs here are grouped together for another reason as well. As a group, they tend to be more powerful (much more so in the case of SYSTAT) than the programs in Group 1, and are consequently unable to fit into the summary table effectively. These are programs well worth considering, and users who demand their flexibiity and capabilities may be willing to forgo the Macintosh interface.

Lionheart Experimental. (List price: \$145. Microsoft BASIC required.) This package consists of a large set of stand-alone programs, written in Microsoft BASIC, to conduct a wide range of analyses in elementary and advanced statistics. The programs, with few exceptions, are locked and cannot be listed or modified by the user. The package has several $t$ tests, and a large number of analysis of variance programs, as well as two substantial multiple regression programs. There are several data preparation programs to handle data for later analysis, but there is no global editor. There is no overall control program to present a consistent user interface, and each program acts independently, offering its own choices by menu. Some programs accept data from an externally prepared file; some also permit direct data entry. Once into a program, the user is guided by menu through the choices necessary to complete the analysis. The documentation accompanying the package consists of $51 / 2 \times 81 / 2$ in. loose-leaf pages (no binder supplied) that are intended to be a textbook on the methods represented by the programs.
The textbook makes no reference to the programs. An appendix describes some of the groups of programs and a few individual programs, identified by using IBM PCtype file names, but not in any detail; the user is left to explore the programs to determine exactly what each one does. There is no list of the names of the programs in the package, and no descriptions of what any program does. For example, there are two programs that, based on their names, look appropriate for one-way analysis of variance. Only by executing them does it become clear that one program handles unequal group sizes, and the other does not. The programs dynamically allocate memory on the Mac, and it is impossible (for even Lionheart's programmer) to determine the exact limits (e.g., numbers of variables, cases, etc.) for each program. Lionheart has put together some very powerful and useful programs in this set; the package might be useful to a computer-sophisticated researcher with the time and patience to explore the programs thoroughly, sufficient memory for detail to remember just what each does, and enough sophistication to know how to make use of them.

Lionheart Business, Forecast, and EDA. (List prices: $\$ 145, \$ 145$, and $\$ 75$, respectively. Microsoft BASIC required.) These are three more sets of BASIC programs. Each shares the general approach to packaging described above. Some of the same programs appear in more than one package, but each set focuses on a group of statistical procedures appropriate to the title of the package.

NWA Statpak 3.15. (List price: \$395. Microsoft BASIC, 512K, and external drive required.) This highly regarded package has been translated for the Macintosh from its $\mathrm{PC}$ and $\mathrm{CP} / \mathrm{M}$ versions; the program is provided in source code, and individual routines can be modified. The main control program and data editor have been rewritten for the Macintosh and offer a combination Mac/PC interface. Once into the operational routines, however, the user is confronted with a mainframe or PC-like screen offering commands and/or menu choices to complete the analysis. The package is highly integrated, and the commands are consistent across all subprograms. Of particular note is the automatic chaining from one subprogram to another. NWA is particuarly good at handling large data files. All data are maintained externally in standard ASCII files, limiting the number of data points only to the capacity of the storage medium. NWA offers a particularly powerful set of regression routines, including both linear and nonlinear procedures, as well as direct, forward, backward, and interactive multiple regression. The analysis of variance features are good, offering one-, two-, and three-way designs, with repeated measures permitted on any or all variables (two- and three-way analyses require equal group sizes). No post hoc analyses are provided. No multivariate methods are included in the package.

SYSTAT. (List price: \$520. Microsoft BASIC, 512K, and external drive required.) Considered the power package for microcomputers, this highly regarded (and very expensive) package brings mainframe capability to the 
desktop. The user is asked to suspend any expectation of a Macintosh interface; it is simply not offered. What is offered is a set of facilities worthy of BMDP, SAS, or SPSS. The program operates through a carefully designed user-command interface. A well-written full-screen data editor offers easy editing even in extremely large data sets. The data handling capabilities are impressive and include SYSTAT's own simplified version of BASIC, which allows the user to write complex data transformation programs, prepare extensive reports, and generate random data. All of the expected descriptive statistics are included in SYSTAT, along with a wide range of graphics options (all printer-style) including not just frequency distributions but also box plots and probability plots. The multivariate general linear hypothesis section allows ANOVA, MANOVA, discriminant analysis, multiple regression, canonical correlation, and multivariate profile analysis of repeated measures. Factor analysis, principal components analysis, cluster analysis, time series procedures, and multidimensional scaling round out the package. This is not a program for students except at advanced levels; this is a full-featured, professional-quality program (and not many professionals will require all of its capabilities). The program is supplied on four diskettes, and is accompanied by an outstanding 400-page manual. The speed (only an IBM PC-AT can run analyses faster, and not by much), capability, and capacity of SYSTAT mark it as the choice for the Macintosh user who insists on having mainframe capability on a Mac and is willing to pay the list price (apparently SYSTAT is not available through discount outlets). Anyone who can afford the price of this program can also probably afford to run it from a hard disk, which will eliminate the disk swapping that is necessary on a two-drive computer.

\section{ACCURACY}

All programs were checked for accuracy using several data sets. All performed acceptably in the descriptive statistics and correlation routines, and on several one- and two-way analysis of variance data sets (which is to say that their results on all computations agreed with known results to the limit of numbers of displayed significant digits).

A frequent area of concern among statistical program reviewers has been accuracy in least squares computations, especially as reflected in multiple regression routines. A very demanding test of computational accuracy employs the notorious Longley (1967) data set. This data set consists of 16 observations on seven economic variables, and is characterized by extremely poorly conditioned data and several highly correlated variables leading to a nearly singular correlation matrix. A test using the Longley data tests primarily the accuracy of a matrix inversion algorithm, since it is the inversion that produces numeric inaccuracies that are reflected in the final regression solution.
SYSTAT, previously shown (Carpenter, Deloria, \& Morganstein, 1984) to be able to not only cope with the Longley data but produce values accurate to 9 significant digits on an IBM PC, served as the benchmark for comparison. Data Desk, StatFast, StatView, StatWorks, and SYSTAT (Macintosh) were tested with the Longley (1967) data; the other programs were not. CLR ANOVA does not do regression; WormStat, MacFits, and Mac.Stat.Pac cannot accommodate seven variables; NWA StatPack was not available in time; and NCSS is about to be released in an entirely different version. StatFast, StatView, StatWorks, SYSTAT Mac, and The Data Desk reproduced SYSTAT IBM's regression coefficients perfectly to the limits imposed by their display format.

The Longley results, which apply only to multiple regression computations, show that the least squares algorithms used in the programs differ considerably in their ability to cope with extremely difficult numerical data. The Longley data, incidentally, are supplied with StatWorks as an example set, showing considerable and warranted confidence in the accuracy of the program.

\section{RECOMMENDATIONS}

The choice of a statistics program for the Macintosh is not easy. After an admittedly slow start (the Mac is difficult to program well), a wide variety of software is now available. A number of the statistics programs reviewed here take full advantage of the Mac's interface and graphics capabilities. Unless there is a need for some feature that is offered by StatFast or NCSS, for example, there is little reason to select a program that does not offer the full Macintosh interface. Among those that do, The Data Desk, StatView, and StatWorks are all excellent choices. All three are good general-purpose programs that are easily learned and used by students and professionals alike. They differ slightly in features (see Table 1), but not appreciably in ease of use. The Data Desk comes with an exceptionally complete manual and a wide variety of interesting data sets, and might be an excellent choice for classroom use, although other features might tip the scale toward some other program. Each of these three programs is soon to be joined by a "professional" version that offers more features, more variables, more analyses, and more ease of use (along with a higher price tag, and requiring a 512K computer). The soon-to-be-released NCSS update may offer another very good possibility.

For an elementary course in statistics, WormStat is an outstanding program that offers the student an easy-touse, intuitive approach to data analysis and exploration. It, too, is soon to be upgraded and should prove more useful.

For analysis of variance, CLR ANOVA is nearly a requirement, although it will not suffice as a general data analysis tool. A combination of CLR ANOVA and one of the general-purpose programs should serve the needs of most behavioral scientists very well. 
Mac.Stat.Pak may appeal particulary to readers of this journal, because of its open invitation to do-it-yourself and contribute to an ongoing program development project. It has much to recommend it as a research tool, as long as the user does not expect too much.

For those who need its power and can afford it, SYSTAT offers a capability and accuracy unmatched among any Mac programs. When using the program, it is easy to forget that you are not connected to a mainframe, except that the results may be obtained much faster. The manual that accompanies SYSTAT is a marvel of clarity, thoroughness, and accuracy, written in a friendly, personal manner that other other manual writers should emulate.
Finally, don't overlook the capabilities of a good spreadsheet/graphics combination. Microsoft's EXCEL is one possibility because of its macro capability, but a combination of Multiplan and Chart (with Switcher) and a good collection of templates can supply many statistical needs.

\section{REFERENCES}

Carpenter, J., Deloria, D., \& Morganstein, D. (1984). Statistical software for microcomputers. Byte, 9(4), 234-264.

LONGLEY, J. W. (1967). An appraisal of least squares programs from the point of view of the user. Journal of the American Statistical Association, $65,819-841$. 